\title{
A numerical model for predicting adsorption behaviour in small activated carbon beds
}

\author{
Samuel G. A. Wood ${ }^{1}$, Nilanjan Chakraborty ${ }^{1}$, Martin W. Smith ${ }^{2}$, Mark J. Summers ${ }^{2}$ \\ ${ }^{1}$ School of Engineering, Newcastle University \\ Newcastle upon Tyne, United Kingdom \\ s.wood8@newcastle.ac.uk \\ ${ }^{2}$ Defence Science and Technology Laboratory \\ Salisbury, United Kingdom
}

\begin{abstract}
A Reynolds-Averaged Navier-Stokes (RANS) model to describe the adsorption of volatile organic compounds on activated carbon beds was developed, using the Dubinin-Radushkevich adsorption isotherm with a Linear Driving Force (LDF) kinetic model. The model was validated using experimental breakthrough data for cyclohexane. The model was then used to predict the impact of adsorption kinetics in small activated carbon beds. The adsorption showed two distinct phases: an initial period where contaminant very rapidly penetrated into the bed, followed by a period where the contaminated front advanced at a slow, constant pace. The initial distance penetrated into the bed was shown to be inversely proportional to the LDF kinetic constant, a trend the same as that predicted using a common plug-flow mass-balance model. The velocity distribution after this initial phase showed some small variations with kinetic constant. This suggests that the kinetic constant may have an impact on the speed of the advance of the contaminated front, an effect not seen in plug-flow models.
\end{abstract}

Keywords: Adsorption, Sctivated carbon, Linear Driving Force, Reynolds Averaged Navier Stokes.

\section{Introduction}

The use of activated carbon as an adsorbent is widespread in industry. A common use is in the treatment of gas streams, in which a flow of contaminated air is passed through an activated carbon bed to provide clean air downstream. The typical mechanism for this process will involve a region of contaminated carbon forming at the inlet of the bed which progresses further through the bed over time when exposed to continuous contaminated flow. Eventually this front will reach the outlet end of the bed, and contaminant will pass through in significant quantities. The time taken for this to occur is known as the breakthrough time of the bed, and represents the useful bed lifetime.

The breakthrough time for large beds is commonly estimated using the Wheeler-Jonas equation, which is derived from a mass balance across a bed under plug flow conditions. The breakthrough time $t_{B}$ for a threshold outlet concentration $c_{\text {out }}$ is expressed as follows [1]:

$$
t_{b}=\frac{\rho_{B} W_{e}}{C_{\text {in }} v_{L}}\left[L-\frac{v_{L}}{k_{v}} \ln \left(\frac{C_{\text {in }}}{C_{\text {out }}}\right)\right]
$$

where there is an inlet velocity $v_{L}$ of gas contaminated with concentration $c_{i n}$ to a bed of nominal density $\rho_{B}$. The overall kinetic constant $k_{v}$ may be described by a number of different empirical or theoretical methods. A common method is to use the following empirical equation [2]:

$$
k_{v}=800 \beta^{0.33} v_{L}^{0.75} d_{p}^{-1.5} \sqrt{\frac{W_{e}}{M_{W}}}
$$


where $\beta$ is an experimentally-assessed affinity coefficient of the target molecule for carbon and $M_{W}$ is its molecular mass, $d_{p}$ is the average carbon particle diameter and $W_{e}$ is the equilibrium uptake of the contaminant on carbon.

The equilibrium uptake for a given condition must be evaluated by use of an appropriate thermodynamic model. Physical adsorption is governed by the attractive potential between the vapour and the porous surface. When vapour is adsorbed, it effectively behaves as a liquid on the carbon surface. This then reduces the adsorption potential until equilibrium is reached for a given temperature and concentration. The choice of thermodynamic adsorption model is dependent on the surface and contaminant.

A widely used semi-empirical model for the adsorption of organic vapours on activated carbon (a common application) is the Dubinin-Radushkevich equation [3].

$$
W_{e}=\rho_{L} W_{0} \exp \left[-\left(\left(\frac{R T}{\beta \mathrm{E}_{\mathrm{o}}}\right) \ln \left(\frac{p_{s a t}}{p_{c}}\right)^{2}\right)\right.
$$

$\rho_{L}$ is the liquid density of the adsorbed vapour, $W_{0}$ is the total micropore volume per unit mass of activated carbon, $\beta E_{o}$ is the adsorption energy, $p_{c}$ is the local partial pressure of contaminant in the vapour phase and $p_{s a t}$ is its saturation pressure.

In many applications, the adsorption kinetics are dictated solely by the intra-particle diffusion rate [4]. It is common to approximate intra-particle diffusion by evaluating the average contaminant concentration over a whole volume using the Linear Driving Force (LDF) approach. The adsorbed uptake of contaminant $W_{t}$ at a given time $t$ is given as [5]:

$$
\frac{W_{t}}{W_{e}}=1-e^{-K_{M} t}
$$

where $K_{M}$ is the effective mass transfer coefficient of the contaminant on carbon.

Although the Wheeler-Jonas equation provides a good estimate of breakthrough times in large carbon beds, it has some limitations [3]. In small beds, the assumption of plug flow will be followed less closely, as the effect of no-slip at the wall becomes more significant. The prediction of an overall kinetic constant $k_{v}$ requires empirical data which may not always be available. It is also limited in use to continuous steady flow rates.

It is therefore desirable to develop a numerical model to better understand the adsorption process in small activated carbon beds. In this study, experimental isotherm and breakthrough data for cyclohexane on activated carbon are used to provide validation for a Computational Fluid Dynamics (CFD) adsorption model. The model is then used to vary the adsorption kinetics to understand its impact on breakthrough time.

\section{Experimental Method}

\section{1. Isotherm Measurements}

The adsorption energy of cyclohexane on activated carbon was found by taking an isotherm measurement using a DVS Advantage from Surface Measurement Systems. A continuous stream of uncontaminated, dry air was passed over a mass ( $25 \mathrm{mg}$ ) of activated carbon. Cyclohexane at a known concentration was then introduced to the stream and the mass change of carbon recorded until no further mass change was seen (the point where equilibrium was attained).

The concentration was then increased and the mass increase measured again. This process was repeated to plot the isotherm over the region of interest.

\section{2. Breakthrough Time Measurements}

A cylindrical carbon bed was used for measuring the breakthrough time. The bed was $15 \mathrm{~mm}$ long and $86.5 \mathrm{~mm}$ in diameter. The activated carbon was packed with a mean particle size of $1 \mathrm{~mm}$ and a nominal density of $550 \mathrm{~kg} \mathrm{~m}^{-3} \mathrm{using}$ the snowstorm technique [6]. 
The clean bed was exposed to a continuous stream of air contaminated with cyclohexane at a concentration of $2000 \mathrm{ppm}$ at $295 \mathrm{~K}$ and the cyclohexane concentration measured at the outlet by means of mass spectrometry.

\section{Numerical Method}

\section{1. Numerical Implementation and Boundary Conditions}

Ansys Fluent [7] was used to solve the coupled mass, momentum, energy and species conservation equations. The equations were discretised using the second order-upwind scheme for the advective terms and second order centraldifferencing scheme for the diffusive terms. A second-order implicit scheme was used for evaluating transient terms. The discretised equations were then solved by use of the SIMPLE algorithm.

The properties of cyclohexane used in this study are given in Table 1.

Table 1: Relevant physical properties of cyclohexane.

\begin{tabular}{|c|c|}
\hline Molecular Weight (kg/kmol) & 84.17 \\
\hline Diffusion Coefficient in air[8] $\left(\mathrm{m}^{2} / \mathrm{s}\right)$ & $7.84 \times 10^{-6}$ \\
\hline Heat of adsorption $(\mathrm{kJ} / \mathbf{k m o l})$ & 18945.5 \\
\hline
\end{tabular}

The density was evaluated using the ideal gas law and diffusion in the gas phase modelled using the Fickian diffusion approximation.

The viscous and inertial resistance of the porous medium was included as a source term for the momentum equations, described as the pressure drop per unit length by the Ergun equation:

$$
(-\Delta \bar{p} / L)=\frac{150 \mu\left(1-E_{p}\right)^{2}}{d_{p}^{2} E_{p}^{3}} v_{s}+1.75 \rho \frac{\left(1-E_{p}\right)}{d_{p} E_{p}^{3}} v_{s}^{2}
$$

where $v_{s}$ is the local superficial fluid velocity, $E_{p}$ is the void fraction and $d_{p}$ is the mean particle diameter.

Within the activated carbon region an additional source term for the mass conservation and species conservation of cyclohexane equations was included using the Dubinin-Radushkevich thermodynamic model with LDF kinetics as described by Eqs. (3) and (4). The heat of adsorption found from the experimental data is included as a source term in the energy equation.

Turbulence was modelled using the $k-\epsilon$ turbulence model with blended wall functions, in which in the fully turbulent region the full transport equations for turbulent kinetic energy $k$ and its dissipation rate $\epsilon$ are solved, whereas in the viscosity affected near-wall region a single equation model is used, and a blending function is used between the two regions.

The computational domain consisted of a cylinder of diameter $86.5 \mathrm{~mm}$ and a depth of $15 \mathrm{~mm}$. $50 \mathrm{~mm}$ of open cylinder were included at the inlet and the outlet.

The inlet velocity was modified dependent on the case. Inlet turbulence was specified as a turbulence intensity $I$ of $10 \%$ and turbulence length scale of $l=0.07 d$ by means of a sensitivity study. The simulation was carried out exploiting the axisymmetry of the domain, such that the gradient of each variable normal to the axis was zero. The walls obeyed the noslip condition. At the outlet boundary the Neumann boundary condition was used (no change in variables in the boundary normal direction).

All simulations were transient, with the time step selected by a sensitivity study as $0.001 \mathrm{~s}$. Clean air was used throughout the domain until no change was seen between time steps and then a mass fraction of $Y_{C_{6} H_{12}}=0.005902$ was introduced at the inlet. The meshes used were investigated for mesh independence by analysing the pressure drop and propagation speed of the contaminated front. 


\section{Results}

\section{1. Experimental Results}

The isotherm data recorded was fitted to the Dubinin-Radushkevich equation as shown in Fig. 1 by selecting values of $\beta E_{o}$.

The model provides a good match to the uptake identified from the experimental results over the range of partial pressures used in this study, although it deviates somewhat very close to the saturation pressure.

The breakthrough times for a $15 \mathrm{~mm}$ cyclohexane at three different flow rates are shown in Table 2.

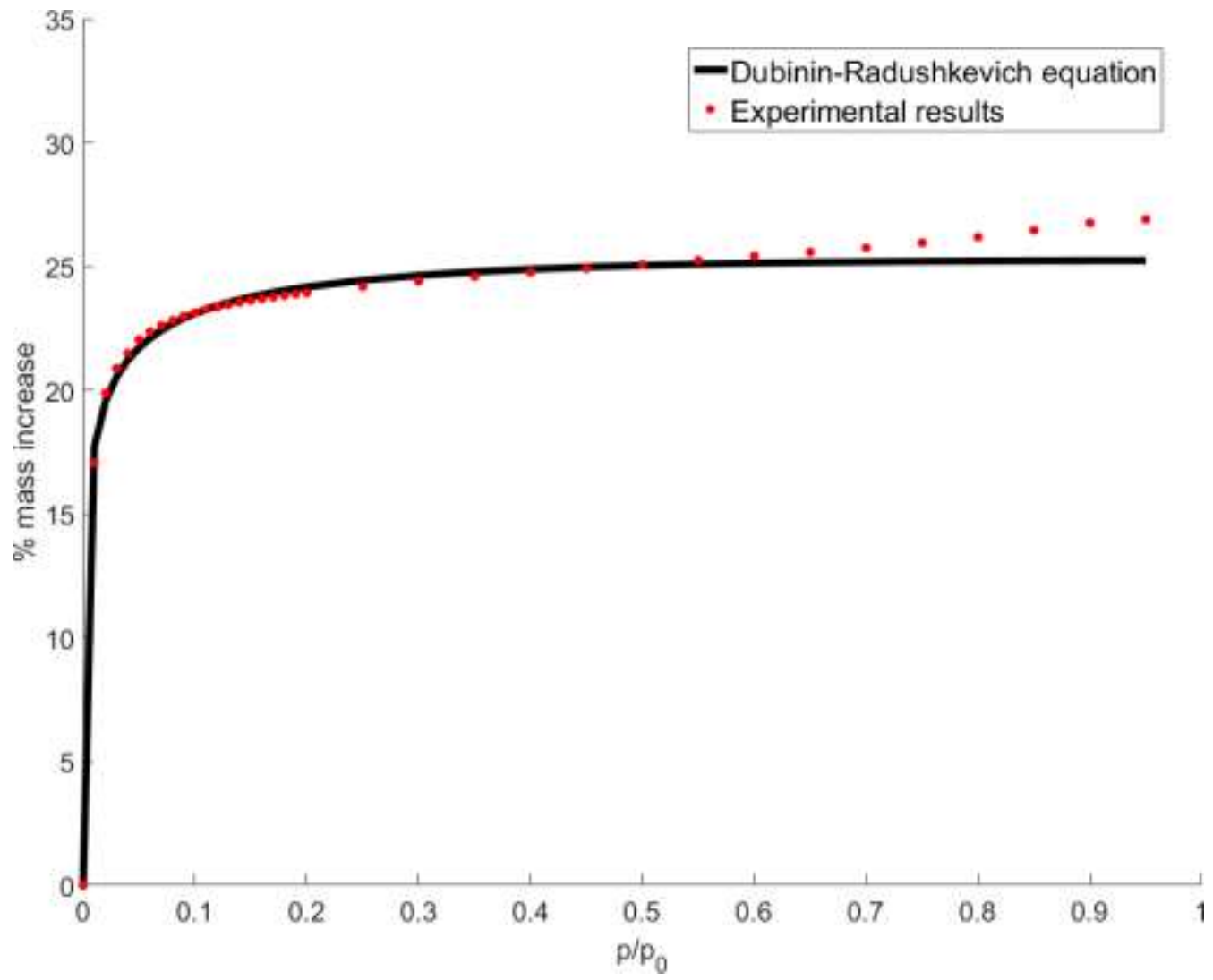

Fig. 1: Adsorption isotherm for cyclohexane on activated carbon at $295 \mathrm{~K}$ compared to the fitted Dubinin-Radushkevich model.

\section{2. Validation}

The CFD model was validated by comparing the breakthrough times of the experimental data with the equivalent CFD calculation. The threshold for breakthrough was considered to be a measurement of $10 \mathrm{ppm}$ at the outlet of the carbon bed.

Table 2: Comparison between time to breakthrough in a $15 \mathrm{~mm}$ bed for different inlet flowrates.

\begin{tabular}{|c|c|c|}
\hline $\begin{array}{l}\text { Flow rate } \\
\left(\mathrm{l} \mathrm{min}^{-1}\right)\end{array}$ & $\begin{array}{l}\text { Experimental } \\
\text { breakthrough time } \\
\text { (s) }\end{array}$ & $\begin{array}{l}\text { CFD } \\
\text { breakthrough } \\
\text { time (s) }\end{array}$ \\
\hline 15 & 5208 & 5010 \\
\hline 30 & 2688 & 2631 \\
\hline 50 & 1407 & 1440 \\
\hline
\end{tabular}


The comparison between CFD results and experimental findings is provided in Table 2. The computational model gave an excellent match to the experimental data.

\section{3. Impact of adsorption kinetics}

The impact of the adsorption kinetics on the adsorption behaviour was analysed by running simulations using five different values of $K_{M}$ (see equation (4)) which were selected as representative of a wide range of volatile organic vapours [9].

The penetration length over time for each of the cases is shown in Fig. 2. The penetration length is defined as the furthest point from the bed inlet that a threshold value of cyclohexane mass fraction could be seen (in this case $Y_{C_{6} H_{12}}=10^{-6}$ was chosen arbitrarily).

It is clear that all cases follow a similar relationship between penetration length and time. An initial period (approximately one second) is seen in which contaminant very rapidly breaks into the bed by a distance which is heavily dependent on the kinetic constant. This is then followed by a period of slow but constant progression of the contaminated region through the bed.

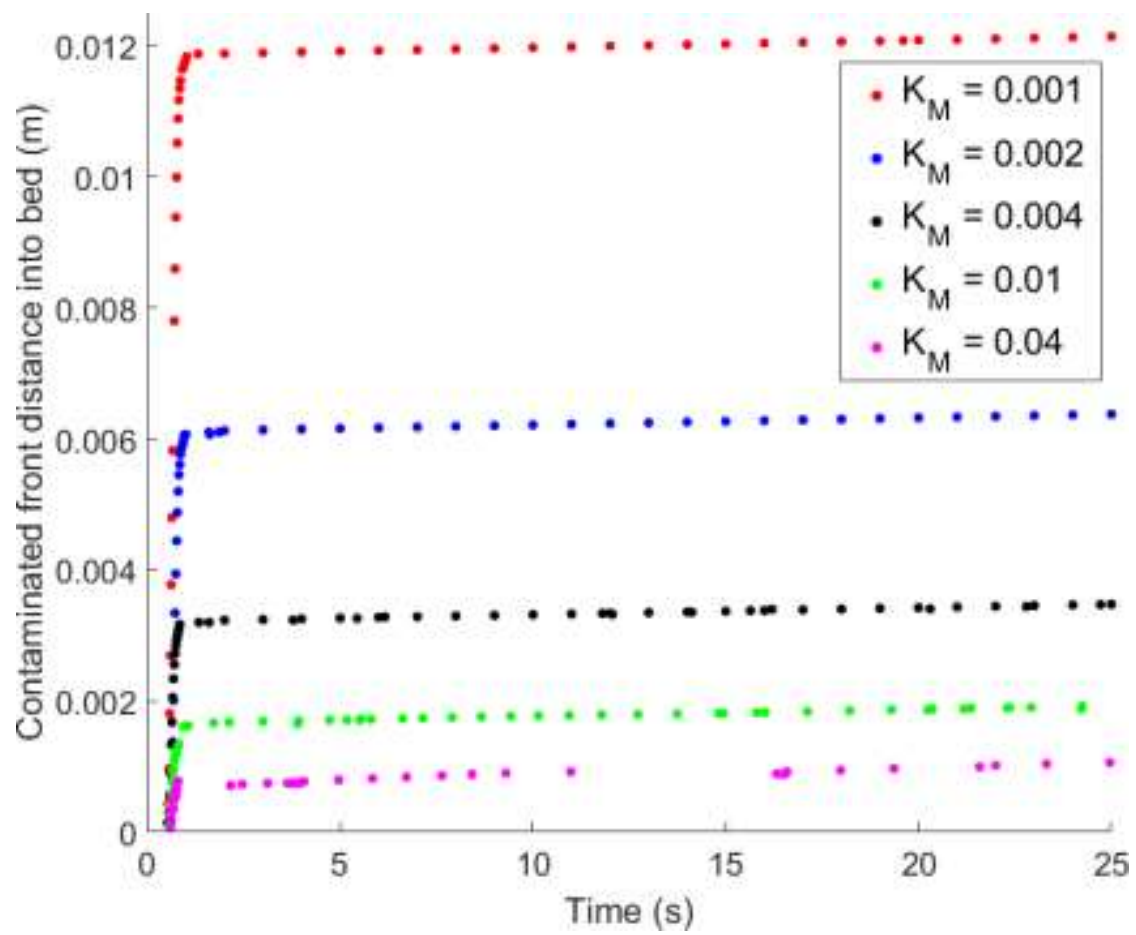

Fig. 2: The distance into the carbon bed that a local mass fraction of greater than $10^{-6}$ can be seen in the bed for different kinetic constants.

The profile can be compared to the Wheeler-Jonas equation, which can be re-written in terms of penetration length $L_{p}$ in the following manner:

$$
L_{p}=\frac{v_{L}}{k_{v}} \ln \left(\frac{C_{\text {in }}}{C_{\text {out }}}\right)+\left(\frac{C_{\text {in }} v_{L}}{\rho_{B} W_{B}}\right)
$$


The Wheeler-Jonas profile predicts that the breakthrough distance can be separated into two components: an initial instantaneous penetration which is inversely proportional to the kinetic constant, and a time-dependent steady increase which is unaffected by kinetics.

The initial length broken into the bed is visualised with respect to the kinetic constant in Fig. 3.

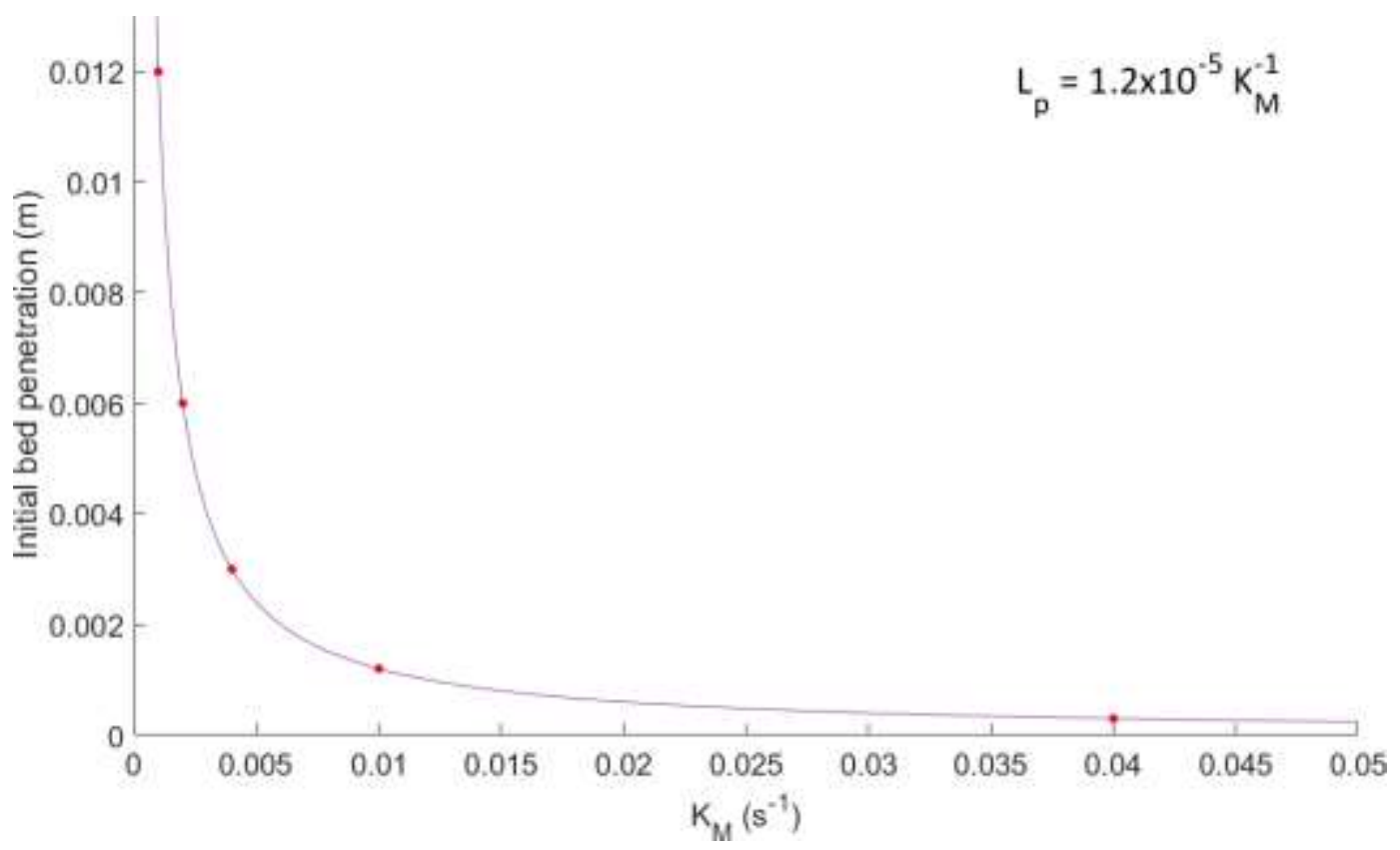

Fig. 3: The initial near-instantaneous penetration into the bed seen using different kinetic constants and the corresponding trend line and equation.

Fig. 3 shows that there is a relationship of inverse proportionality between the initial penetration distance and the kinetic constant, similar to that seen using the Wheeler-Jonas equation. The Wheeler-Jonas equation predicts that this behaviour is instantaneous. Although this is clearly unrealistic, the time taken to reach this length is near-constant in all cases and is very small compared to the speed of the contaminated front after the initial penetration. 


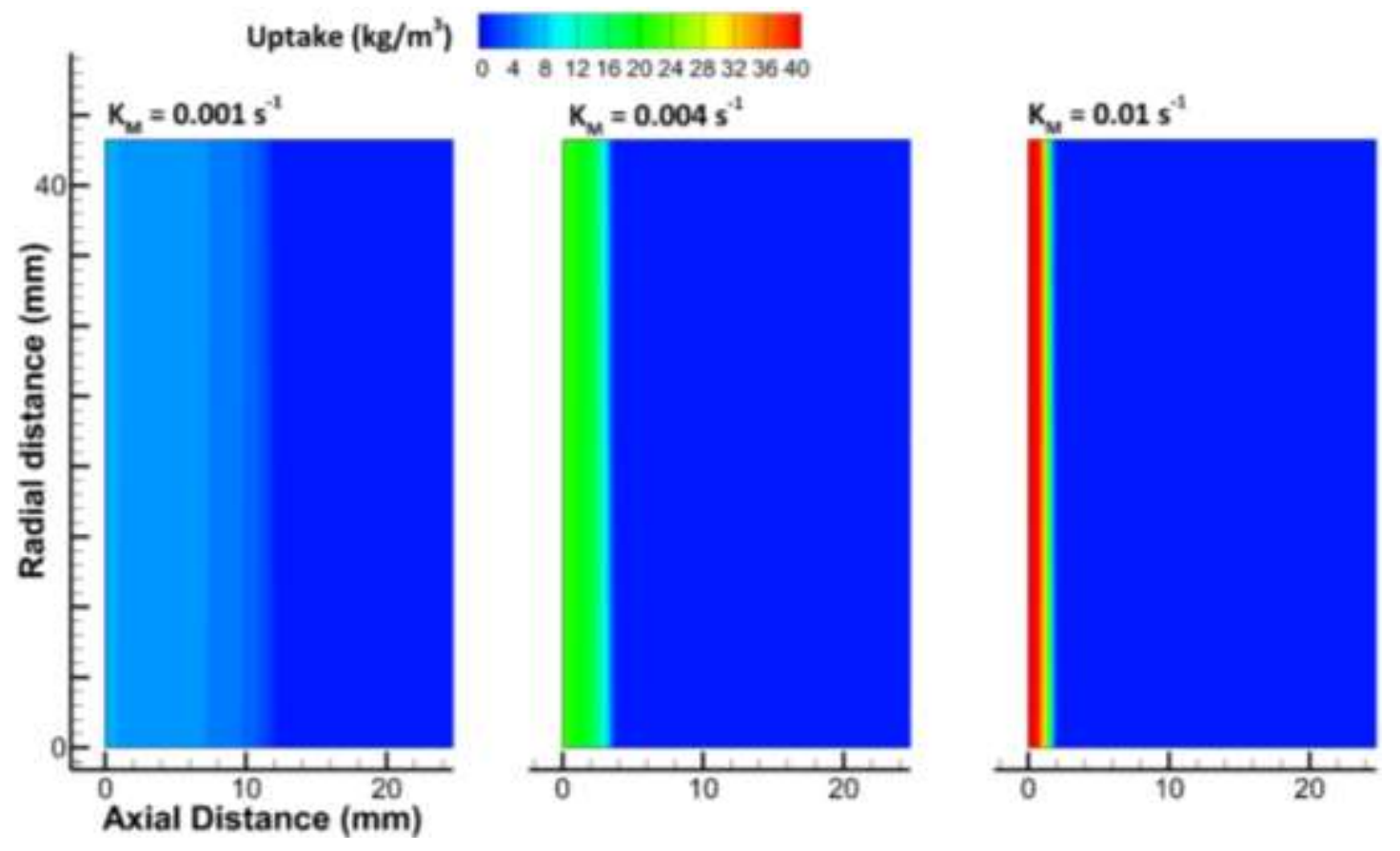

Figu. 4: The distribution of contaminant uptake at $60 \mathrm{~s}$ after initial exposure for different values of $K_{M}$.

After this initial period the front advances at a constant speed. Under plug-flow conditions Eq. 6 suggests that this front speed should be dependent on the rate at which contaminant enters the bed and also on the adsorption capacity of the bed, while independent of kinetics.

Inspection of Fig. 4 shows that after identical time spent after initial exposure, the adsorbed contaminant varies in how it is distributed throughout the bed. As contaminant is adsorbed, there will be a small resultant reduction in fluid volume, which will reduce the velocity. Two example velocity distributions are shown in Fig. 5.

The time-dependent term in the Wheeler-Jonas equation indicates that the speed of the front after the initial stage of rapid penetration into the bed will be independent of kinetics. However, inspection of the axial velocity distributions reveals that there is some relationship between kinetics and fluid velocity, which may affect the front speed. 

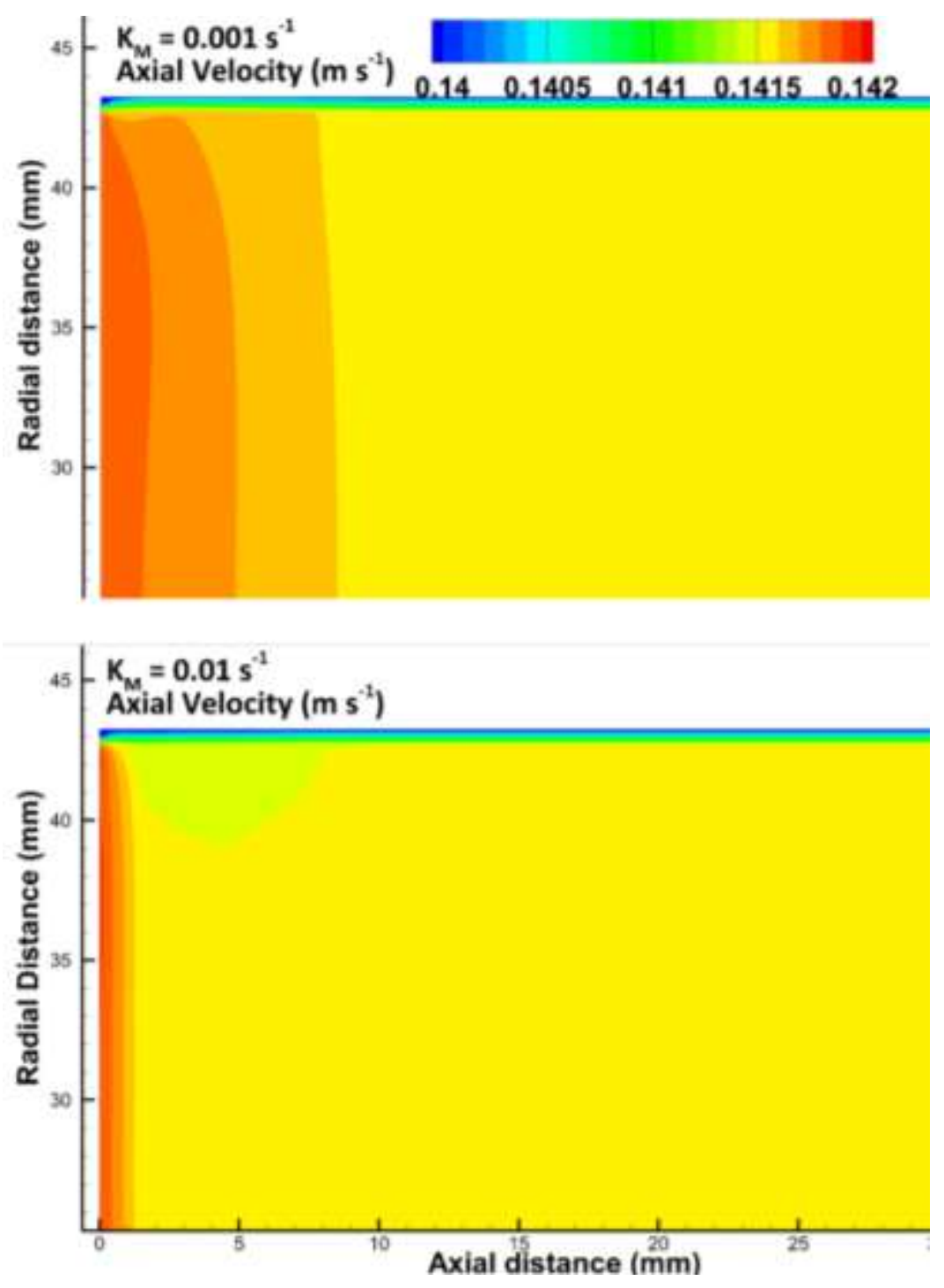

Fig. 5: Axial velocity distribution close to the canister wall at the inlet of the carbon bed 10 seconds after the initial introduction of contaminant for two different kinetic constants.

\section{Conclusions}

Experiments were conducted to measure the adsorption isotherm of cyclohexane onto granular activated carbon and the breakthrough time for various flowrates of cyclohexane-contaminated air through a small activated carbon bed. Data from these experiments were then used to develop a CFD model to predict this adsorption behaviour using the DubininRadushkevich thermodynamic model limited by Linear Driving Force kinetics. The CFD model showed an excellent match to the experimental data.

The CFD model was used to predict variations in adsorption behaviour based on varying the kinetic constant of the LDF model. In all cases, the penetration into the bed was a two stage process, with an initial near-instantaneous distance governed by the kinetics and a continuous time-dependent distance thereafter.

The kinetically governed initial penetration distance was found to be inversely proportional to the LDF kinetic constant, a similar trend to that seen when using the Wheeler-Jonas equation. 
For the same exposure time and total uptake, different kinetic constants resulted in different distributions of adsorbed contaminant throughout the bed and thus different velocity profiles. This suggests that the extent to which different contaminants deviate from the plug flow assumption may vary. Consequently there may be some impact of adsorption kinetics on the front speed after the initial stage of rapid penetration into the bed, which will be the subject of future investigations.

\section{Acknowledgements}

The authors are grateful to EPSRC, UK, for financial support.

\section{References}

[1] L. A. Jonas and J. A. Rehrmann, "The kinetics of adsorption of organo-phosphorus vapors from air mixtures by activated carbons," Carbon, vol. 10, pp. 657-663, 1972.

[2] G. O. Wood and P. Lodewyckx, "An Extended Equation for Rate Coefficients for Adsorption of Organic Vapors and Gases on Activated Carbons in Air-Purifying Respirator Cartridges," AIHA Journal, vol. 64, pp. 646-650, 2003.

[3] P. Lodewyckx, G. O. Wood, and S. K. Ryu, "The Wheeler-Jonas equation: a versatile tool for the prediction of carbon bed breakthrough times," Carbon, vol. 42, pp. 1351-1355, 2004.

[4] T. S. Y. Choong and D. M. Scott, "The linear driving force model for cyclic adsorption and desorption: the effect of external fluid-film mass transfer," Chemical engineering science, vol. 53, pp. 847-851, 1998.

[5] C. R. Reid and K. M. Thomas, "Adsorption Kinetics and Size Exclusion Properties of Probe Molecules for the Selective Porosity in a Carbon Molecular Sieve Used for Air Separation," The Journal of Physical Chemistry B, vol. 105, pp. 10619-10629, 2001.

[6] E. Harris, "Improvements in or relating to the filling of granular materials into containers," United Kingdom Patent 606, 867, 1946.

[7] ANSYS, Academic Research, Release 16.2: Ansys, 2016.

[8] A. N. Berezhnoi and A. V. Semenov, Binary diffusion coefficients of liquid vapors in gases. New York: Begell House, 1997.

[9] A. J. Fletcher, Y. Yuzak, and M. Thomas, "Adsorption and desorption kinetics for hydrophilic and hydrophobic vapors on activated carbon," Carbon, vol. 44, pp. 989-1004, 2006. 